\title{
OBTURATION OF ROOT CANALS BY VERTICAL CONDENSATION OF GUTTA-PERCHA - BENEFITS AND PITFALLS
}

\section{CALKOVSKY BRUNO, SLOBODNIKOVA LADISLAVA, BACINSKY MARTIN, JANICKOVA MARIA}

Clinic of Stomatology and Maxillofacial Surgery, Jessenius Faculty of Medicine in Martin, Comenius University in Bratislava, and Martin University Hospital, Martin, Slovakia

\begin{abstract}
A b s tract
Vertical condensation of gutta-percha is one of the frequently used techniques of hermetic and three-dimensional obturation of root canal system which is one of the primary goals of endodontic tooth treatment. Techniques for filling root canals have been described since ancient Egypt and have undergone dynamic development in recent decades. The aim of the article is to analyze the technique of obturation of root canals using vertical condensation of guttapercha and to point out its benefits and pitfalls.

The poor-quality treatment of the tooth deteriorates its prognosis, function, and long-term survival in the oral cavity; thus, the hermetic obturation of the root canals is one of the most important pillars of the complex tooth treatment. Obturation of the root canals by vertical condensation of warm gutta-percha is nowadays considered in the correct indications as one of the most commonly used techniques in the excellent dental practices. Even though it is quite technologically challenging and time-consuming it enables to reach high level of predictability and clinical success. Currently, the obturation technique based on master gutta-percha cone combined with the calciumsilicate based sealer is emerging. However, long term results of clinical studies and follow-up are needed to make a significant improvement in the area of endodontics.
\end{abstract}

Keywords: endodontics, vertical condensation, gutta-percha, root canal obturation

\section{INTRODUCTION}

The dental pulp may be irritated by various pathological processes with bacterial pathogens from progressive dental caries, periodontal lesions, or micro/macro trauma of the tooth being most common. Disruption of physiological defense mechanisms leads to inflammation or necrosis of the dental pulp with possible harm to the periapical area and potential development of abscess or phlegmon (Farges et al., 2015). According to the prognosis of the tooth and the overall condition of the patient the dentist decides between the endodontic and extraction therapy (Hargreaves and Berman, 2016).

The methods of root canals obturation has undergone significant development. The root canals were already filled by ancient Chinese or Egyptians. The concept and understanding of endodontic treatment changed throughout the history and is changing dynamically nowadays as well. In the near past, diverse techniques and materials have been used for obturation. In Slovakia, the technique of conventional root canal filling with a central gutta-percha cone was preferred. Recently, in accordance with the introduction of modern devices and materials, obturation using a central gutta-percha cone in combination with the calcium si-

Corresponding author: Assoc. Prof. MUDr. Maria Janickova, PhD., MPH; e-mail: maria.janickova@uniba.sk (C) 2021 Janickova M. et al.

This work is licensed under the Creative Commons Attribution-NonCommercial-NoDerivs 4.0 License (https://creativecommons.org/licenses/by-nc-nd/4.0/) 
licate sealer or complete obturation of the canals with calcium silicate materials has come to the fore (Zare et al., 2021). The technique of obturating root canals by vertical condensation of gutta-percha is one of the leading techniques and has proven to be the most suitable in certain clinical indications.

The correct choice of the obturation technique is crucial. A variety of available materials as well as instrumentation systems make the decision difficult. Moreover, the root systems may have complicated anatomy, which makes the right choice even more challenging. Vertical condensation of gutta-percha or its combination with other techniques represents the optimal option in most clinical situations with a potential of high-quality treatment result.

Despite the fact that we can predictably make a long-lasting root filling nowadays, it is necessary to realize that the best "filling" of the root canal is its healthy dental pulp. For this reason, it has to be always preserved and its iatrogenic damage must be avoided.

\section{Obturation of root canals - equipment and materials}

Endodontic devices enabling vertical condensation of gutta-percha have appeared for the first time in the 1960s as a novelty representing an efficient way of obturating the root canals. Over the past, this technology has undergone significant development, improving its properties and significantly eliminating the negatives and risks it entails.

A significant milestone in the development was the introduction of the "backfill" tool which allows the exact and fast placement of the plastic gutta-percha only by pressing and holding a button. Combining the use of a heatplugger (also known as a "DownPack") and a backfill, these devices represent a significant leap in technology and have quickly become used by dentists all around the world (Fig.1) (Perry et al., 2013).

Gutta-percha is an inert material with a relatively good biocompatibility and cytotoxic properties described in literature are attributed to the zinc oxide it contains. As gutta-percha cones have a different composition, their toxicity is also different. However, it is still one of the least cytotoxic materials of its kind available on the market. Unlike most sealer mate-

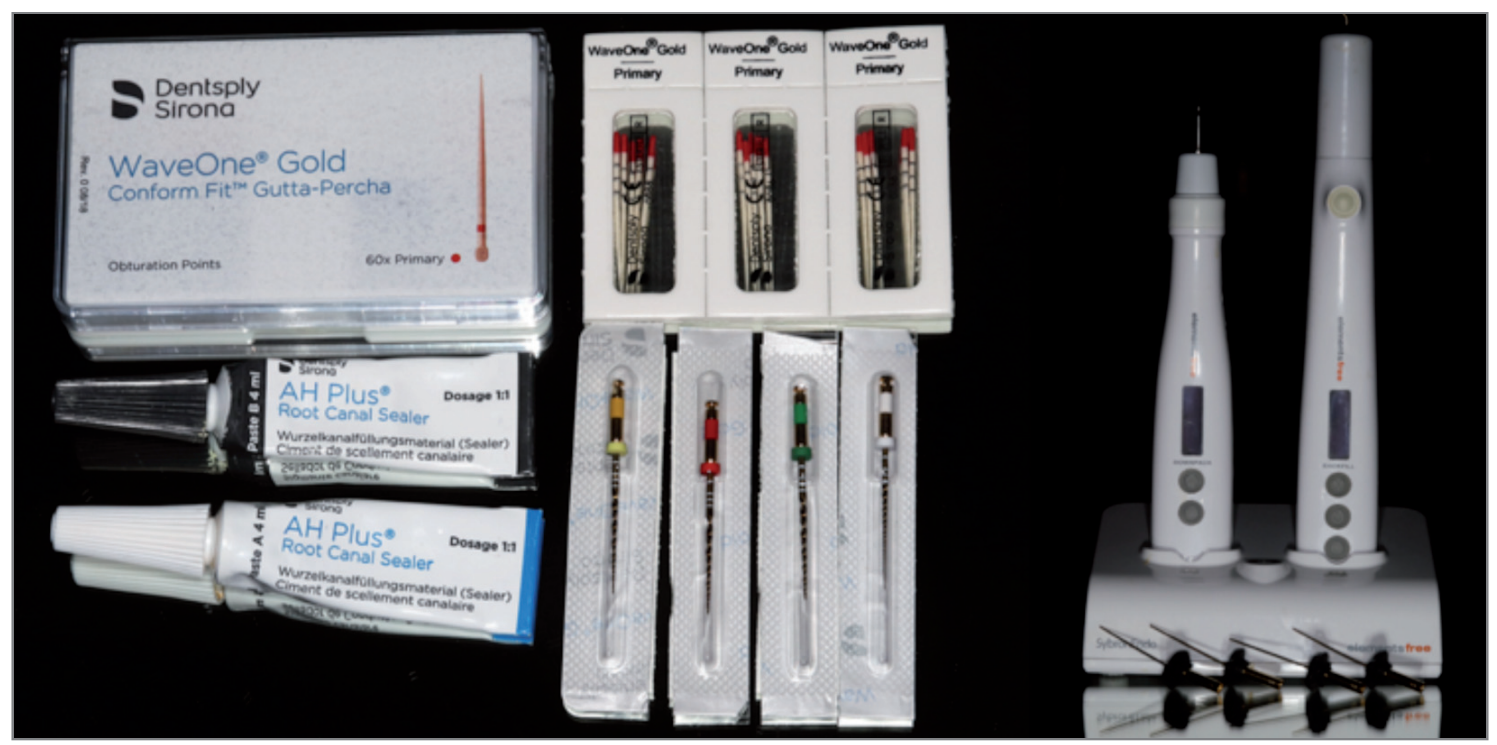

Fig.1 Equipment and materials. Gutta-percha cones, paper points, WaveOne Gold endodontic files, AH Plus sealer (Dentsply Sirona) and Elements Free (SybronEndo). (Source: author) 
rials, gutta-percha is non-resorbable. Its extrusion behind the apex of the tooth is in most cases associated with postoperative complications.

Gutta-percha is soluble in organic solvent which are used to remove it from the root canals during re-endodontic treatment. Effective gutta-percha solvents include e.g. chloroform or eucalyptol (Dotto et al., 2021). It does not adhere to the dentin of the root system and, therefore, all filling techniques using gutta-percha cones require their combination with sealer material.

In dentistry, there are several forms of available gutta-percha but for the needs of vertical condensation it is only necessary to characterize conventional gutta-percha cones and thermoplastic gutta-percha in the form intended for injection (Fig. 1). This form of gutta-percha is produced as pellets which are introduced into a heating system (Obtura III - Obtura) or as prepared cannulas (ElementsTM, Free-Kerr). The injection of a thermoplastic gutta-percha itself is a separate technique, but its use - the name of the "backfill" technique in combination with a central gutta-percha cone - is the basis of the gutta-percha vertical condensation technique.

\section{Comparison of individual techniques of root canal obturation}

The correct choice of the obturation technique with regard to the peculiarities of the treated tooth is very important. At the same time, the dentist's skills and experience as well as the use of modern equipment such as magnifying glasses or operating microscope play a role. In addition to the technique mentioned herein other frequently used methods include the obturation technique of lateral condensation of cold gutta-percha, the technique of applying gutta-percha using a carrier, and filling the root canal in its entirety with calcium silicate materials (e.g., MTA - mineral trioxide aggregate) (Vadachkoria et al., 2019).

Post-endodontic treatment, whether direct or indirect filling or crown, isolates the root system with filling from the environment of the oral cavity. In the event of failure of this barrier, the root filling is exposed to saliva and bacteria.

The root system is considerably complicated and often asymmetric. It may complicate the achievement of a high-quality three-dimensional hermetic seal. In each of the filling techniques tested, a leakage of different extent was demonstrated between the root filling and the canal wall. The leakage rate is related to the resistance of the root filling and insufficient isolation of post-endodontic treatment.

The leakage of root fillings made by vertical condensation of gutta-percha, lateral condensation of cold gutta-percha, and Thermafill technique was evaluated in seventy extracted single-rooted teeth which were decapitated at the cementoenamel junction (Gilbert et al., 2001). Following the extraction, the teeth were stored for 90 days in 100\% humidity and after this period the penetration depth of Proteus vulgaris and methylene blue along the fill was monitored over time. Proteus vulgaris was chosen because of its similar size to endodontic pathogens and ease of cultivation. In contrast to previous studies with "India Ink" dye the authors used methylene blue the molecules of which are of a size comparable to endodontic pathogens. The methylene blue leakage was evaluated using the stereomicroscopic pictures at 6x magnification of cleared sample teeth and their examination. The leakage was evaluated and measured with a ruler $(0,5$ increments) by two independent examiners according to a specific scoring system (Gilbert et al., 2001).

The most perfect seal among the three techniques was achieved with the gutta-percha vertical condensation technique, followed by the Thermafill technique. The most significant leakage was found in the cold gutta-percha lateral condensation technique. As leakage has been reported with each of the technique, it is appropriate to consider the need for root canal re-treatment in root filling exposed to the oral environment (Mathur et al., 2015).

The anatomy of the root system is complex and may be even more complicated by various resorptive pathological processes. The lacuna formed by the process of internal resorption 
represents an extreme asymmetry in the root canal. It creates a taper that can be problematic to fill with incorrectly chosen root fill technique. Gencoglu with co-authors (2008) compared several obturation techniques using gutta-percha in filling of an artificially created resorptive lacuna in the middle third of the root canal. They evaluated the degree of filling at the widest point of the lacuna at the horizontal section through the canal and the ratio of sealer material in the root filling. Although the study did not compare the technique of vertical condensation of gutta-percha, the advantage of obturation of root canals by guttapercha heated and condensed in the root canal in a given anatomical situation was clearly demonstrated. Cold gutta-percha obstruction and Thermafill techniques have proven unsatisfactory.

The three-dimensional obturation of the roots is preceded by its disinfection and mechanical-chemical preparation in order to simplify the root system and by chemical preparation of lateral ramifications and dentinal tubules. Chemical-mechanical preparation and disinfection is very important, but no less is their hermetic obturation. In in vitro study the filling of the lateral ramifications by the method of lateral and vertical condensation of the gutta-percha were compared.

The authors demonstrated that with lateral condensation it is not possible to obturate the lateral ramifications. Vertical condensation enables it, especially in the coronary and middle third of the root canal. Filling of the lateral ramifications $1 \mathrm{~mm}$ from the apex was infrequent (Wolf et al., 2021).

\section{Risk factors of the obturation technique}

In the technique of vertical condensation of gutta-percha three main risk factors have been identified. They can lead to a damage of periodontium during treatment and prevent or slow down the healing process of the periapical area. The first one is mainly a vertical condensing pressure which increases the probability of excessive extrusion of the obturating material into the periapical space. The second factor is associated with the condensing pressure as well. It may be a cause of dentinal crack propagation leading to a vertical root fracture when obturating the root canal. The third factor is a relatively high temperature of the tip of the heatplugger in the root canal, which can damage the surrounding structures.

\section{Filling materials extruded into periodontium}

As it is a non-resorbable material, the extrusion of a gutta-percha cone poses a risk to the healing of periapex. Any pushing of the gutta-percha into this area should be avoided. In contrast to gutta-percha, there are several opinions on the extrusion of a small amount of sealer material (Meneses et al., 2019). One of the preconditions of successful endodontic treatment is the filling of the root canal in its entire length up to the apical constriction. Pushing of a small amount of sealer material into the periodontium corresponds to the shading in the periapical space visible on the X-ray image and is known as "puff" (Fig. 2).

In the root canal, which has not been obturated and, above all, disinfected up to the apical constriction, microorganisms or necrotic pulp residues may remain and trigger pathological changes.

In the following section, the endodontic sealer AH Plus (Dentsply Sirona) is discussed as it is one of the frequently used sealer materials, especially with the method of vertical condensation of gutta-percha (Ricucci et al., 2016).

Sealer materials with different properties are available on the market. With respect to their interaction with periodontal tissues, the degree of cytotoxicity or biocompatibility, solubility, and irritation of periapical tissues is important. For several sealer materials (e.g. AH Plus, Dentsply) their cytotoxic properties are also mentioned, which, however, are present only during solidification of the material. In this particular case, it is about 48 hours, which is not essential in clinical practice (Ricucci et al., 2016). 
Not every extruded sealer material is completely resorbed within four years. Sealer material AH Plus (Dentsply) belongs to the category of frequently used sealers that contain epoxy resin. An X-ray examination revealed a complete resorption of extruded AH Plus material in only $15 \%$ of cases within four years (Ricucci et al., 2016).

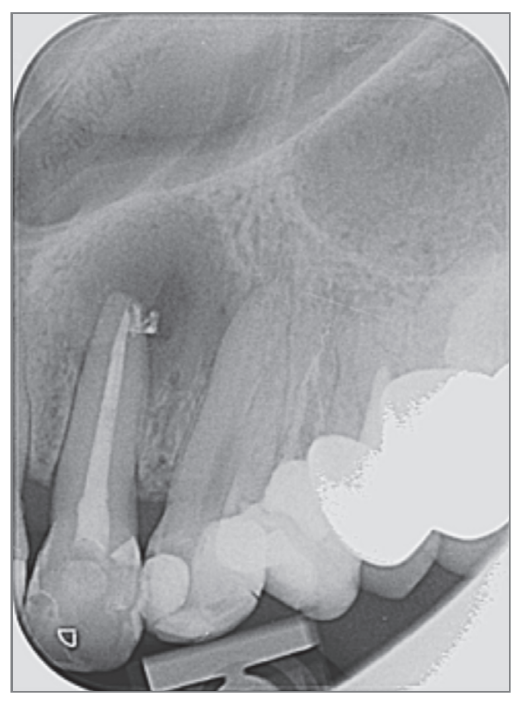

Fig. 2 Postoperative X-ray after obturation by the method of vertical condensation of gutta-percha. The "puff" of the sealer AH Plus (Dentsply Sirona) can be seen on the upper lateral incisor. (Source: author)

\section{Dentinal crack propagation}

In different methods of obturation, a certain condensation pressure is applied to the walls of the root canal. If this pressure is too high, cracks formed in the dentin can lead to a vertical fracture of the tooth. In particular, the thickness of the dentin wall gives the resistance of the root to condensing pressures. Therefore, it is an important factor that also has to be taken into the account when selecting the technique and performing the obturation. A suitable way to prevent vertical root fractures in endodontic treatment is a non-aggressive root canal preparation and an adequate condensation pressure in obturation (Chai and Tamse, 2018).

When comparing methods of vertical condensation, lateral condensation, and injection gutta-percha obturations based on the comparison of micro CBCT images it was found that the highest propagation of cracks in the dentin was with lateral condensation of gutta-percha (Chellapilla et al., 2021).

\section{Influence of temperature on periodontium}

The tip of a heatplugger (e.g. ElementsTM Free; Kerr) develops an intraradicular temperature of up to $300{ }^{\circ} \mathrm{C}$. Long exposure to such a high temperature can lead to a necrosis of periodontal cells. The manufacturer's declared temperature of $200{ }^{\circ} \mathrm{C}$ is developed only on the tip of the heatplugger. It is preceded by a short discharge of $300{ }^{\circ} \mathrm{C}$, which accelerates the heating of the tip. The tip which is the largest source of the heat is isolated from periodontium by gutta-percha, sealer, or dentin of the root canal. This significantly reduces the amount of heat that acts on the periodontium. Dentin as well as gutta-percha are considered very good thermal insulators. For periodontal tissues, an increase in the temperature of more than $10{ }^{\circ} \mathrm{C}$ above the physiological temperature is critical and can lead to a thermal tissue injury (Eriksson and Albrektsson, 1983).

Sweatman and coauthors (2001) tested in vitro the effect of thermoplastic gutta-percha temperature on periodontal temperature. The procedure recommended by the manufacturer 
increased the tissue temperature by less than $10^{\circ} \mathrm{C}$ in either case. Failure to follow a proper procedure can lead to an increase in temperature in the periodontium and a potential thermal injury (Sweatman et al., 2001).

It is not possible to relate the results of the study to in vivo conditions. E.g., the circulation of blood in the periodontium may be protective as it dampens the effect of the applied temperature on the periodontium (Cen et al., 2018).

\section{CONCLUSIONS}

The right choice of the obturation technique does not suffice to reach a predictably successful therapy. The decisive factors that affect the patient's outcome are the knowledge, skill, and experience of the dentist. Last but not least, endodontic treatment requires plenty of time and the need to focus. All above-mentioned factors serve as preconditions for a successful endodontic therapy

The technique of vertical condensation of gutta-percha represents, in the correct indication, the optimal way of obturation of root canals. By following the manufacturer's recommendations and workflow the risks associated with this technique can be eliminated and a successful therapy can be reached.

Conflict of Interest: The authors deny any conflicts of interest related to this study.

\section{REFERENCES}

1. Capar ID, Saygili G, Ergun H, Gok T, Arslan H, Ertas H. Effects of root canal preparation, various filling techniques and retreatment after filling on vertical root fracture and crack formation. Dent Traumatol. 2015;31(4):302-7.

2. Cen R, Wang R, Cheung GSP. Periodontal Blood Flow Protects the Alveolar Bone from Thermal Injury during Thermoplasticized Obturation: A Finite Element Analysis Study. J Endod. 2018 Jan; 44(1):139-144.

3. Chai H, Tamse A. Vertical Root Fracture in Buccal Roots of Bifurcated Maxillary Premolars from Condensation of Gutta-percha. J Endod. 2018 ;44(7):1159-1163.

4. Chellapilla PK, Boddeda MR, Jyothi M, Uppalapati LV, Konagala RK, Dasari L. Influence of obturating techniques on root dentin crack propagation: A micro-computed tomography assessment. J Conserv Dent. 2021;24(1):72-76.

5. Dotto L, Sarkis-Onofre R, Bacchi A, Pereira GKR. The use of solvents for gutta-percha dissolution/removal during endodontic retreatments: A scoping review. J Biomed Mater Res B Appl Biomater. 2021;109(6):890-901.

6. Eriksson AR, Albrektsson T. Temperature threshold levels for heat-induced bone tissue injury: a vital-microscopic study in the rabbit. J Prosthet Dent 1983; 50(1): 101-107.

7. Farqes JC, Alliot-Licht B, Renard E, Ducret M, Gaudin A, Smith AJ, Cooper PR. Dental Pulp Defence and Repair Mechanisms in Dental Caries. Mediators Inflamm. 2015; 2015:230251.

8. Gencoglu N, Yildirim T, Garip Y, Karagenc B, Yilmaz H. Effectiveness of different gutta-percha techniques when filling experimental internal resorptive cavities. Int Endod J 2008; 41(10): 836-842.

9. Gilbert SD, Witherspoon DE, Berry CW. Coronal leakage following three obturation techniques. Int Endod J 2001; 34(4): 293-299.

10. Hargreaves KM, Berman LH. Cohen's Pathways of the Pulp. 11. edition, St.Louis, Missouri: Elsevier, 2016, 964 s. ISBN-13: 978-0323096355

11. Mathur R, Sharma M, Sharma D, Raisingani D, Vishnoi S, Singhal D, Grover S. Evaluation of coronal leakage following different obturation techniques and in-vitro evaluation using methylene blue dye preparation. J Clin Diagn Res 2015; 9(12): ZC13-17. 
12. Meneses CB, Gambini AF, Olivi LT, Santos MD, Sipert CR. Effect of CPoint, EndoSequence BC, and Gutta-percha Points on Viability and Gene Expression of Periodontal Ligament Fibroblasts. Eur Endod J. 2019; 4(2):57-61.

13. Perry C, Kulild JC, Walker MP. Comparison of warm vertical compaction protocols to obturate artificially created defects in the apical one-third. J Endod. 2013;39 (9)

14. Ricucci D, Rôças IN, Alves FR, Loghin S, Siqueira JF Jr. Apically extruded sealers: fate and influence on treatment outcome. J Endod 2016; 42(2): 243-249.

15. Sweatman TL, Baumgartner JC, Sakaguchi RL. Radicular temperatures associated with thermoplasticized gutta-percha. J Endod 2001; 27(8): 512-515.

16. Vadachkoria O, Mamaladze M, Jalabadze N, Chumburidze T, Vadachkoria D. Evaluation of three obturation techniques in the apical part of root canal. Georgian Med News. 2019 ;(292-293):17-21.

17. Wolf TG, Willems L, Briseño-Marroquín B. An in vitro endodontic model to quantify the accessory canal filling potential of the vertical and lateral condensation techniques. Aust Endod J. 2021; 47(2):245-251.

18. Zare S, Shen I, Zhu Q, Ahn C, Primus C, Komabayashi T. Micro-computed tomographic evaluation of single-cone obturation with three sealers. Restor Dent Endod. 2021;46 (2):e25.

Received: October,25,2021

Accepted: November,20,2021 